\title{
Leucovorin rescue allows effective high-dose pralatrexate treatment and an increase in therapeutic index in mesothelioma xenografts
}

\author{
Philip M. Tedeschi • Yamini K. Kathari • \\ Iqra N. Farooqi · Joseph R. Bertino
}

Received: 17 July 2014 / Accepted: 30 August 2014 / Published online: 9 September 2014

(C) The Author(s) 2014. This article is published with open access at Springerlink.com

\begin{abstract}
Purpose To investigate the ability of leucovorin (LV) to abrogate dose-limiting toxicities of pralatrexate (PDX) while maintaining efficacy, in vivo.

Methods H2052 mesothelioma cells were treated with the antifolates methotrexate (MTX), PDX and pemetrexed, with and without LV rescue $24 \mathrm{~h}$ later. Cell killing was evaluated $48 \mathrm{~h}$ later. Female nude mice bearing H2052 xenografts were treated with varying doses and schedules of the antifolate PDX and LV.

Results In vitro, $\mathrm{H} 2052$ cells were more sensitive to PDX as compared to MTX and pemetrexed. Administration of LV $24 \mathrm{~h}$ after antifolate treatment reduced efficacy of antifolates MTX and pemetrexed, but not PDX. In vivo, LV was found to reduce toxicity of PDX at the maximum tolerated dose without sacrificing efficacy. Lethal doses of PDX were rescued by LV, and mice bearing the H2052 tumor demonstrated prolonged and enhanced tumor regression.

Conclusions High-dose PDX with subsequent LV rescue may be a viable treatment strategy in mesothelioma and other cancers. The inclusion of LV rescue into new and existing PDX treatment protocols should be explored as a way to expand the tolerability and effectiveness of PDX in the clinic.
\end{abstract}

Keywords Pralatrexate $\cdot$ Antifolate $\cdot$ Mesothelioma . Leucovorin $\cdot$ Folinic acid

P. M. Tedeschi · Y. K. Kathari · I. N. Farooqi · J. R. Bertino ( $₫)$ Departments of Pharmacology and Medicine, Rutgers Cancer Institute of New Jersey, Rutgers, The State University of New Jersey, 195 Little Albany Street, New Brunswick, NJ, USA e-mail: bertinoj@cinj.rutgers.edu

\section{Introduction}

Folic acid and derivatives (folates) are required by mammalian cells to carry out one-carbon transfer reactions and the de novo synthesis of nucleic acids [1]. Folate cannot be synthesized de novo and must be consumed. Once internalized, folic acid is reduced by dihydrofolate reductase (DHFR) to tetrahydrofolate, which then can act to deliver one carbon units to acceptor molecules. Antifolates target rapidly cycling cancer cells, whose enhanced need of one carbon units for de novo pyrimidine and purine synthesis leaves them vulnerable to DHFR inhibition [2]. Aminopterin, a DHFR inhibitor, was the first antifolate used in the clinic, but it fell out of favor when less toxic methotrexate (MTX) was introduced in the 1950s [3]. MTX continues to be included in many therapeutic regimens today.

Pralatrexate (PDX) is a second generation antifolate recently approved for the treatment of peripheral T-cell lymphoma [4]. In addition to inhibiting DHFR, PDX also is an excellent substrate for folylpolyglutamate synthase (FPGS) which polyglutamylates folates, leading to greater cellular retention and activity, and the reduced folate carrier-1 (RFC-1), the primary folate membrane transporter [5]. These additional PDX properties that increase PDX transport and retention in cancer cells lead to improved efficacy when compared to MTX [6].

Adverse events associated with antifolates can be severe, commonly mucositis and leukopenia. A strategy to avoid toxicity and still provide clinical benefit is the inclusion of leucovorin (LV) in high-dose MTX regimens [7]. $\mathrm{LV}$ is a stable, reduced form of folate that is converted to tetrahydrofolate without requiring DHFR, thus bypassing the inhibition of tetrahydrofolate synthesis by MTX and PDX. LV also competes for binding with antifolates at the 


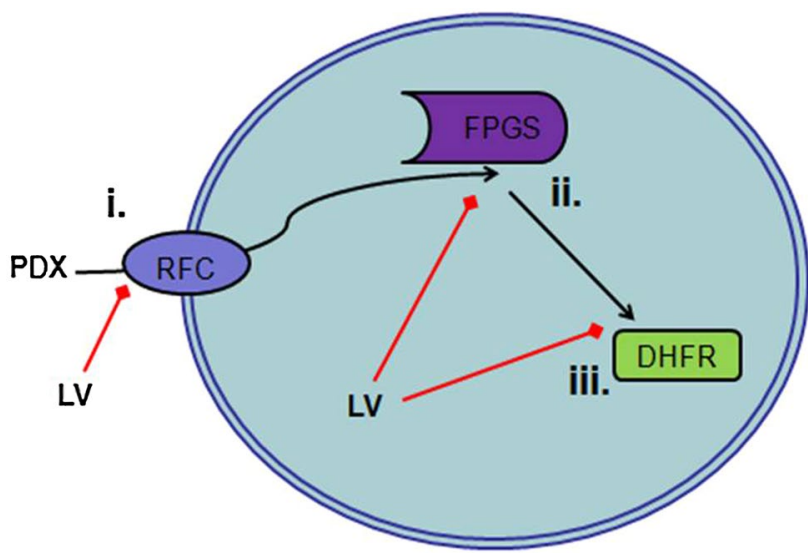

Fig. 1 Leucovorin (LV) may abrogate pralatrexate (PDX) activity through three mechanisms. $i$ Competition for reduced folate carrier type 1 (RFC) transport into cell. ii Competition for polyglutamylation, a retention and activity marker, by folylpolyglutamate synthase (FPGS). iii Provides an alternate source of tetrahydrofolate, working around PDX inhibition of dihydrofolate reductase (DHFR)

reduced folate carrier, and when converted to tetrahydrofolate, competes with antifolates for polyglutamylation (Fig. 1).

In this study, we demonstrate the protective effect of LV on PDX toxicity and show that a significant antitumor effect is achievable with high-dose PDX and LV rescue in mesothelioma xenografts.

\section{Materials and methods}

In vitro cytotoxicity

Five thousand H2052 pleural mesothelioma cells per well were plated in 96-well plates in RPMI 1640 media (Gibco) supplemented with $10 \%$ dialyzed FBS (Invitrogen). The following day, spent media was removed and fresh media containing drug was added and plates were incubated for $24 \mathrm{~h}$. Drug containing media was removed and media containing $2 \mu \mathrm{M} \mathrm{LV}$ was added and plates were incubated for $24 \mathrm{~h}$. LV containing media was removed and fresh media was added to the plates incubated for $48 \mathrm{~h}$. The Cell Titer 96 Aqueous One Solution (Promega) assay was used to assess cell viability at the end of the experiment according to manufacturers' protocol. Data were analyzed using the GraphPad Prism 6 software package (GraphPad Software Inc.).

In vivo experiments

Eight- to 10-week-old female nude mice (Taconic) were subcutaneously injected on the right flank with
5 million $\mathrm{H} 2052$ cells in a 1:1 mixture of matrigel (BD Biosciences) and PBS (Gibco). Mice were monitored until palpable tumors developed and reached a size of $100 \mathrm{~mm}^{3}$. Mice were then randomized and split into the following treatment groups: saline control; 60 or $180 \mathrm{mg} / \mathrm{kg}$ PDX on days 1,4 and $7 ; 60$ or $180 \mathrm{mg} / \mathrm{kg}$ PDX on days 1,4 and 7 followed by $50 \mathrm{mg} / \mathrm{kg} \mathrm{LV} \mathrm{24,}$ 32 and $48 \mathrm{~h}$ after each PDX administration; $50 \mathrm{mg} / \mathrm{kg}$ LV following the above schedule. General toxicity was monitored through weight loss. Tumor dimensions were measured every 2-4 days using calipers, and tumor volume was calculated using the following equation: Volume $=\left(\right.$ width $\left.^{2}\right) \times($ length/2). All animal experiments were approved by the Rutgers Institutional Animal Care and Use Committee.

\section{Results}

To assess the sensitivity to antifolate therapy, a comparison of MTX, PDX and pemetrexed in the pleural mesothelioma cell line $\mathrm{H} 2052$ was performed. After a $24 \mathrm{~h}$ treatment with drug, the media was removed and replaced with fresh media and cultured a further $72 \mathrm{~h}$. PDX demonstrated significant efficacy over MTX in this assay, with $\mathrm{IC}_{50}$ values of 0.625 and $80 \mathrm{nM}$, respectively (Fig. 2a, b). The large difference between PDX and MTX is likely due to enhanced transport and retention of PDX. H2052 cells were also more sensitive to PDX than pemetrexed (Fig. 2c), an antifolate currently used in the clinic to treat mesothelioma.

As LV can abrogate antifolate toxicity, we next examined if efficacy of PDX would be compromised by the addition of LV $24 \mathrm{~h}$ later. H2052 cells were treated with antifolate as before, but media containing $2 \mu \mathrm{M} \mathrm{LV}$ was added for another $24 \mathrm{~h}$ period, followed by removal of drugs and culture in fresh media for a further $48 \mathrm{~h}$. The addition of $2 \mu \mathrm{M} L V$ raised the $\mathrm{IC}_{50}$ slightly for MTX and pemetrexed (Fig. 2d). The effect of LV on tumor cell sensitivity to PDX was only at high PDX concentrations, and was not expected to be significant in in vivo xenografts. We predicted that using PDX in combination with LV rescue would result in an increased therapeutic index.

The effect of LV treatment on PDX efficacy and toxicity was examined using $\mathrm{H} 2052$ xenografts in nude mice (Fig. 3). Using $60 \mathrm{mg} / \mathrm{kg}$ as the equivalent clinical dose, tumor regression was seen both with and without LV treatment in this cohort. Efficacy was nearly identical, but mice that were treated with LV had less toxicity and bodyweight remained close to control animals.

To mimic high-dose MTX treatment with LV rescue, using PDX, we included a cohort treated at $180 \mathrm{mg} / \mathrm{kg}$ 


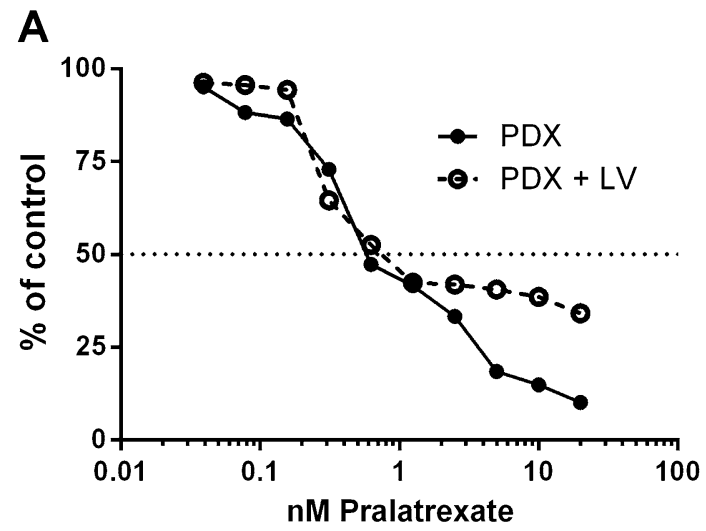

C

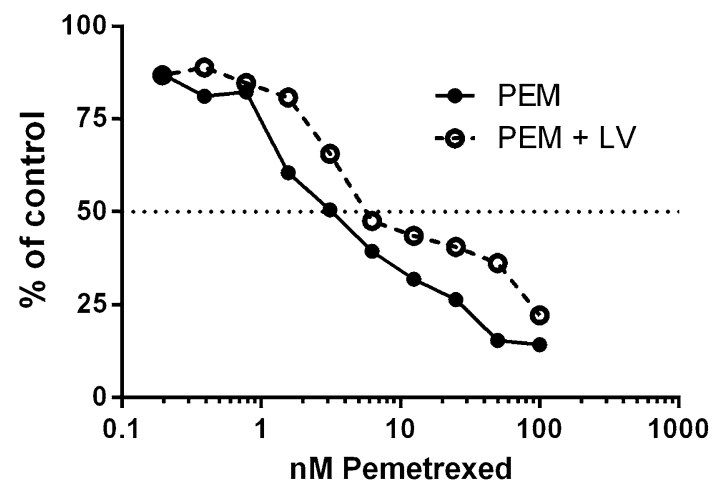

Fig. 2 Pralatrexate (PDX) is more potent than methotrexate and pemetrexed in vitro. Dose response curves shown as percent of viable H2052 mesothelioma cells remaining after $24 \mathrm{~h}$ treatment with pralatrexate (a), methotrexate (b) or pemetrexed (c) followed by $72 \mathrm{~h}$ cul-

PDX with and without LV rescue. Without LV rescue, mice lost a large amount of weight, had poor BCS scores and had to be killed according to institutional protocol (Fig. 3b). Mice rescued with LV exhibited some weight loss, and a durable response lasting several weeks was observed (Fig. 3a).

\section{Discussion}

Surgical resection followed by chemotherapy is the preferred method of treatment for malignant mesothelioma [9]. The antifolate pemetrexed is currently used alone and in combination with cisplatin in treating mesothelioma, with modest response rates seen in the clinic [10]. Chemotherapy is primarily palliative in mesothelioma and there is a need for new and innovative therapies that have the ability to improve patient outcomes [11].

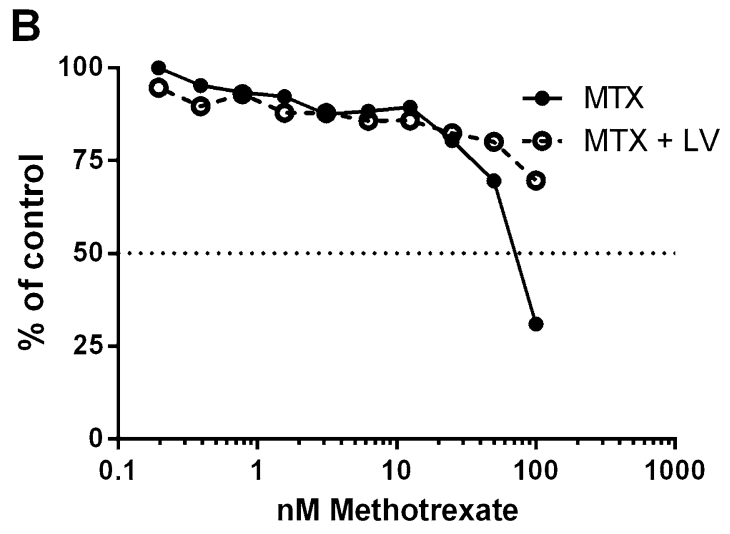

D

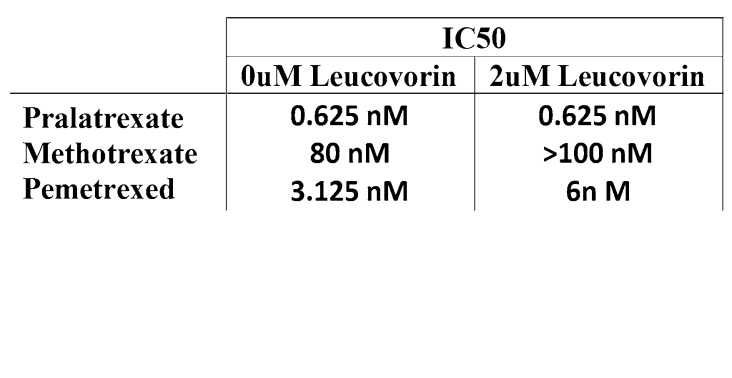

ture in fresh media (solid line) or followed by $24 \mathrm{~h}$ culture in $2 \mu \mathrm{M}$ leucovorin and $48 \mathrm{~h}$ culture in media (dashed line). d A summary table of IC50 values

There were no responses to PDX in a phase II study in mesothelioma [12]. To improve tolerability, PDX was administered at $135 \mathrm{mg} / \mathrm{m}^{3}$ biweekly instead of weekly, possibly limiting clinical effectiveness. A dose schedule of PDX weekly with LV rescue may demonstrate improved antitumor activity with tolerable toxicity. Supporting this conclusion, a small recent case series exploring LV rescue and PDX in T-cell cutaneous lymphoma found that LV rescue allowed patients to tolerate PDX therapy while preserving efficacy [8]. Dose scheduling must be optimized in patients, as the interval between antifolate and LV administration has recently been shown to be significant in determining the efficacy of therapy in vitro [13].

The incorporation of LV rescue with current PDX dosing regimens may prove an effective method to prevent DLTs and increase the therapeutic index. While a phase II study of PDX in patients with mesothelioma did not show responses, higher doses with the inclusion of $\mathrm{LV}$ rescue 


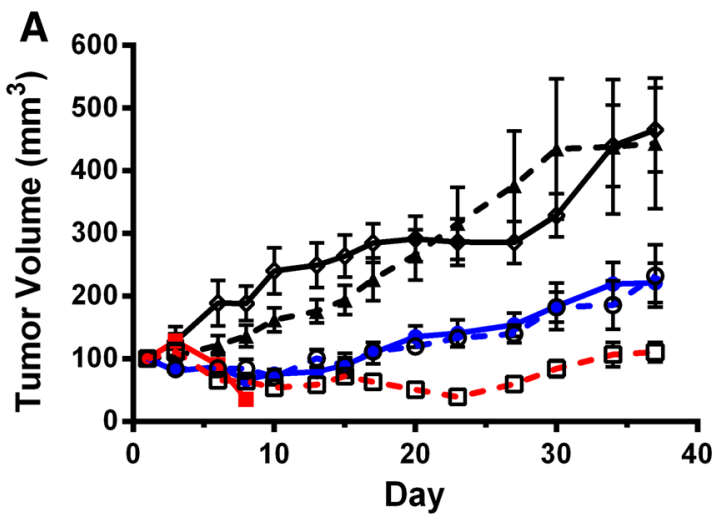

B

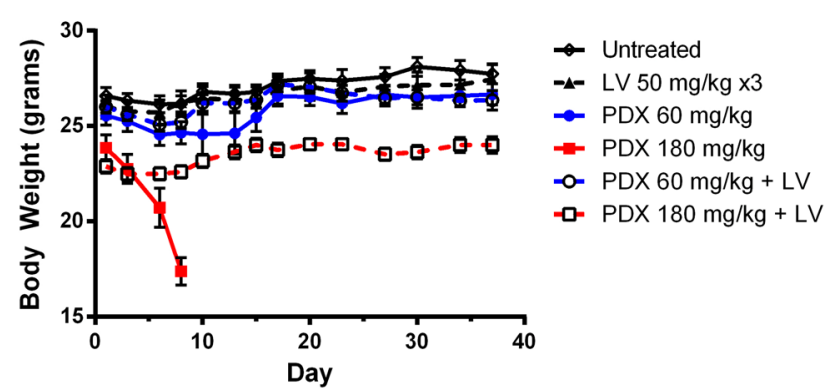

Fig. 3 A and B Pralatrexate is effective against H2052 xenografts. Mice bearing $100 \mathrm{~mm}^{3}$ tumors were treated by i.p injection of saline control; 60 or $180 \mathrm{mg} / \mathrm{kg}$ PDX on days 1,4 and $7 ; 60$ or $180 \mathrm{mg} /$ $\mathrm{kg}$ PDX on days 1,4 and 7 followed by $50 \mathrm{mg} / \mathrm{kg}$ leucovorin 24,32 and $48 \mathrm{~h}$ after each PDX administration; $50 \mathrm{mg} / \mathrm{kg}$ leucovorin. Tumor volume (a) and body weight (b) were measured

into new and existing PDX treatment protocols should be explored as a way to expand the tolerability and effectiveness of PDX in the clinic.

Acknowledgments This study was supported by the National Institutes of Health Biotechnology Training Program (PT) and a grant from Spectrum Pharmaceuticals, Inc (Irvine, CA).

\section{Conflict of interest None.}

Open Access This article is distributed under the terms of the Creative Commons Attribution License which permits any use, distribution, and reproduction in any medium, provided the original author(s) and the source are credited.

\section{References}

1. McGuire JJ, Hsieh P, Coward JK, Bertino JR (1980) Enzymatic synthesis of folylpolyglutamates. Characterization of the reaction and its products. J Biol Chem 255:5776-5788

2. Kamen BA, Nylen PA, Camitta BM, Bertino JR (1981) Methotrexate accumulation and folate depletion in cells as a possible mechanism of chronic toxicity to the drug. Br J Haematol 49:355-360. doi:10.1111/j.1365-2141.1981.tb07237.x

3. Johns DG, Iannotti AT, Sartorelli AC, Bertino JR (1966) The relative toxicites of methotrexate and aminopterin. Biochem Pharmacol 15:555-561

4. Visentin M, Unal ES, Zhao R, Goldman ID (2013) The membrane transport and polyglutamation of pralatrexate: a new-generation dihydrofolate reductase inhibitor. Cancer Chemother Pharmacol 72:597-606. doi:10.1007/s00280-013-2231-9

5. Marchi E, Mangone M, Zullo K, O’Connor OA (2013) Pralatrexate pharmacology and clinical development. Clin Cancer Res Off J Am Assoc Cancer Res 19:6657-6661. doi:10.1158/1078-0432.CCR-12-2251

6. Izbicka E, Diaz A, Streeper R et al (2009) Distinct mechanistic activity profile of pralatrexate in comparison to other antifolates in in vitro and in vivo models of human cancers. Cancer Chemother Pharmacol 64:993-999. doi:10.1007/s00280-009-0954-4

7. Hryniuk WM, Bertino JR (1969) Treatment of leukemia with large doses of methotrexate and folinic acid: clinical-biochemical correlates. J Clin Invest 48:2140-2155

8. Koch E, Story SK, Geskin LJ (2013) Preemptive leucovorin administration minimizes pralatrexate toxicity without sacrificing efficacy. Leuk Lymphoma 54:2448-2451. doi:10.3109/10428194 .2013.779688

9. Ahmed I, Ahmed Tipu S, Ishtiaq S (2013) Malignant mesothelioma. Pak J Med Sci 29:1433-1438

10. Boons CCLM, VAN Tulder MW, Burgers JA et al (2013) The value of pemetrexed for the treatment of malignant pleural mesothelioma: a comprehensive review. Anticancer Res 33:3553-3561

11. Neumann V, Löseke S, Nowak D et al (2013) Malignant pleural mesothelioma: incidence, etiology, diagnosis, treatment, and occupational health. Dtsch Ärztebl Int 110:319-326. doi:10.3238 /arztebl.2013.0319

12. Krug LM, Heelan RT, Kris MG et al (2007) Phase II trial of pralatrexate (10-propargyl-10-deazaaminopterin, PDX) in patients with unresectable malignant pleural mesothelioma. J Thorac Oncol Off Publ Int Assoc Study Lung Cancer 2:317-320. doi:10. 1097/01.JTO.0000263715.84567.5f

13. Visentin M, Unal ES, Goldman ID (2014) The impact of 5-formyltetrahydrofolate on the anti-tumor activity of pralatrexate, as compared to methotrexate, in HeLa cells in vitro. Cancer Chemother Pharmacol 73:1055-1062. doi:10.1007/s00280-014-2441-9 